\title{
A Novel Method for Preparation of Carrageenan/Fish Scale Collagen/Allopurinol Biocomposite Film
}

\author{
Quoc Manh Vu (D), ${ }^{1}$ Thuy Chinh Nguyen $\mathbb{D i}^{1,2}$ Duong My Ngoc Dam, ${ }^{3}$ Quoc Trung Vu $\left(\mathbb{D},{ }^{4}\right.$ \\ Trong Lu Le, ${ }^{2}$ Tran Dung Hoang $\mathbb{D}^{2}$, Thi Kim Ngan Tran $\mathbb{D}^{1,5}$ Tuan Anh Nguyen $\mathbb{D}^{6}{ }^{6}$ \\ Phi Hung Nguyen, ${ }^{7}$ and Hoang Thai ${ }^{1,2}$ \\ ${ }^{1}$ Graduate University of Science and Technology, Vietnam Academy of Science and Technology, 18 Hoang Quoc Viet, Cau Giay, \\ Hanoi 100000, Vietnam \\ ${ }^{2}$ Institute for Tropical Technology, Vietnam Academy of Science and Technology, 18 Hoang Quoc Viet, Cau Giay, \\ Hanoi 100000, Vietnam \\ ${ }^{3}$ Nam Dinh University of Nursing, 257 Han Thuyen, Nam Dinh 420000, Vietnam \\ ${ }^{4}$ Faculty of Chemistry, Hanoi National University of Education, No. 136 Xuan Thuy Road, Cau Giay District, \\ Hanoi 100000, Vietnam \\ ${ }^{5}$ NTT Institute of High Technology, Nguyen Tat Thanh University, 300A Nguyen Tat Thanh, District 4, \\ Ho Chi Minh City 700000, Vietnam \\ ${ }^{6}$ Faculty of Chemical Technology, Hanoi University of Industry, No. 298, Cau Dien Street, Bac Tu Liem District, \\ Hanoi 100000, Vietnam \\ ${ }^{7}$ Institute of Natural Products Chemistry, Vietnam Academy of Science and Technology, 18, Hoang Quoc Viet, Cau Giay, \\ Hanoi 100000, Vietnam
}

Correspondence should be addressed to Thuy Chinh Nguyen; thuychinhhn@gmail.com and Hoang Thai; hoangth@itt.vast.vn

Received 23 March 2021; Revised 6 June 2021; Accepted 23 June 2021; Published 20 July 2021

Academic Editor: Ulrich Maschke

Copyright ( 2021 Quoc Manh Vu et al. This is an open access article distributed under the Creative Commons Attribution License, which permits unrestricted use, distribution, and reproduction in any medium, provided the original work is properly cited.

\begin{abstract}
Biopolymers such as carrageenan or collagen can be used as carriers for loading a drug to enhance a drug's bioavailability. In this work, allopurinol was loaded on a carrageenan/collagen blend and the carrageenan/collagen/allopurinol (CCA) biocomposite films were prepared using the ionic gelation method combined with the $3 \mathrm{D}$ printing method using carrageenan/collagen/allopurinol gel as a $3 \mathrm{D}$ printing ink material. The advantages of the $3 \mathrm{D}$ printing method are the ease in shaping the design of films and the ease in controlling the thickness of films. The results of infrared (IR) spectroscopy and field emission scanning electron microscopy (FESEM) analyses showed that the CCA biocomposite films have a regular structure, and the functional groups of components in the biocomposites can interact with each other. After 30 minutes of immersion in distilled water and $\mathrm{pH}$ buffer solution, the biocomposite films swelled and disintegrated. The carrageenan/collagen blend can control the release of allopurinol in simulated body fluids. In addition, the drug release kinetic models reflecting the release process of allopurinol from CCA biocomposite films in simulated body fluids have also been calculated.
\end{abstract}

\section{Introduction}

In recent years, natural biopolymers and their biocomposites have been of great interest as effective drug carriers to develop potential biopolymer-based drug delivery systems for controlled drug delivery and enhanced drug bioavailability thanks to their biocompatibility, nontoxicity, biodegrad- ability, stability, high drug loading efficacy, and renewable nature. Moreover, owing to the variety of their structures, the differences of their physiological functions, and their distinct properties, they can provide a range of biomedical applications [1-4]. Many natural biopolymers have been assessed for their suitability as a matrix for drugs, including chitosan, alginate, xanthan gum, carrageenan, collagen, 
gelatin, and protein. Among them, collagen and carrageenan have been chosen as suitable biomaterials for the fabrication of polymeric drug delivery systems [4-7].

Collagen has been known as a drug carrier with many advantages such as nontoxicity, high biocompatibility, and good interactions with drugs $[4,5,8,9]$. Collagen has been used alone or in combination with other polymers (chitosan, polycaprolactone, carboxymethyl chitosan, hyaluronic acid, etc.) for loading a number of drugs such as antibacterial drugs, anti-inflammatory drugs, tobramycin or ciprofloxacin, doxorubicin, and gentamicin. [10-23]. Carrageenan, a sulfated polysaccharide obtained from seaweed, has been also recognized as an effective matrix for drug delivery [6, 7, 24-33].

In this study, carrageenan has been combined with collagen for drug delivery thanks to the formation of ionic interactions and hydrogen bonding between carrageenan (an anionic polymer) and collagen (a protonated polymer) [33]. The carrageenan-collagen polyelectrolyte complex can improve the stability, strength, drug loading, and drug release control ability of the carrageenan/collagen blend. Moreover, carrageenan has a good gelling ability and swelling ability, and this can help to enhance the solubility of a drug in the drug release process from a matrix $[6,7]$. Collagen in this case has been extracted from fresh water fish scales. This would promote the utilization of fish wastes during fish processing. Allopurinol-a poorly water-soluble drug-is chosen as a model drug for the carrageenan/collagen blend. Allopurinol and its active metabolite, oxipurinol, inhibit the enzyme xanthine oxidase and prevent the conversion of hypoxanthine and xanthine precursors into uric acid so that blood uric acid levels decrease to help treat gout and other bone-joint disease $[25,34,35]$. It has poor bioavailability after ingestion (a half-life of 1 hour) [25]; therefore, using natural polymers as a matrix for an allopurinol system is necessary. In our previous report, carrageenan/collagen hydrogel beads are suitable for loading allopurinol. The solubility of allopurinol was increased by 1.5 to 6.7 times when loaded on carrageenan/collagen hydrogels [36].

During the past half century, almost all the material processing technologies have tried their potential application in solving the issue of poor water solubility [37]. These technologies include chemical methods such as self-assembly; physical methods such as electrospinning, electrospraying, and $3 \mathrm{D}$ printing; and also the combination of these methods [38-41]. The drug-containing polymeric films are suggested as promising controlled release systems [42-46]. These films can be fabricated by solvent casting, solution casting, or extrusion. The combination of the ionic gelation method and the $3 \mathrm{D}$ printing method to fabricate the carrageenan/fish scale collagen films loading the allopurinol drug which is a combination of chemical and physical methods has had limited studies in the literature. By ionic gelation method, the carrageenan/collagen/allopurinol systems can be obtained in a gel and considered as a 3D printing ink. The design and thickness of films have been controlled by using the $3 \mathrm{D}$ printing method. The time for drying films at room temperature is fast. Therefore, this study focuses on the evaluation of some properties of carrageenan/collagen/allopurinol biocomposite films which were prepared by the ionic gelation

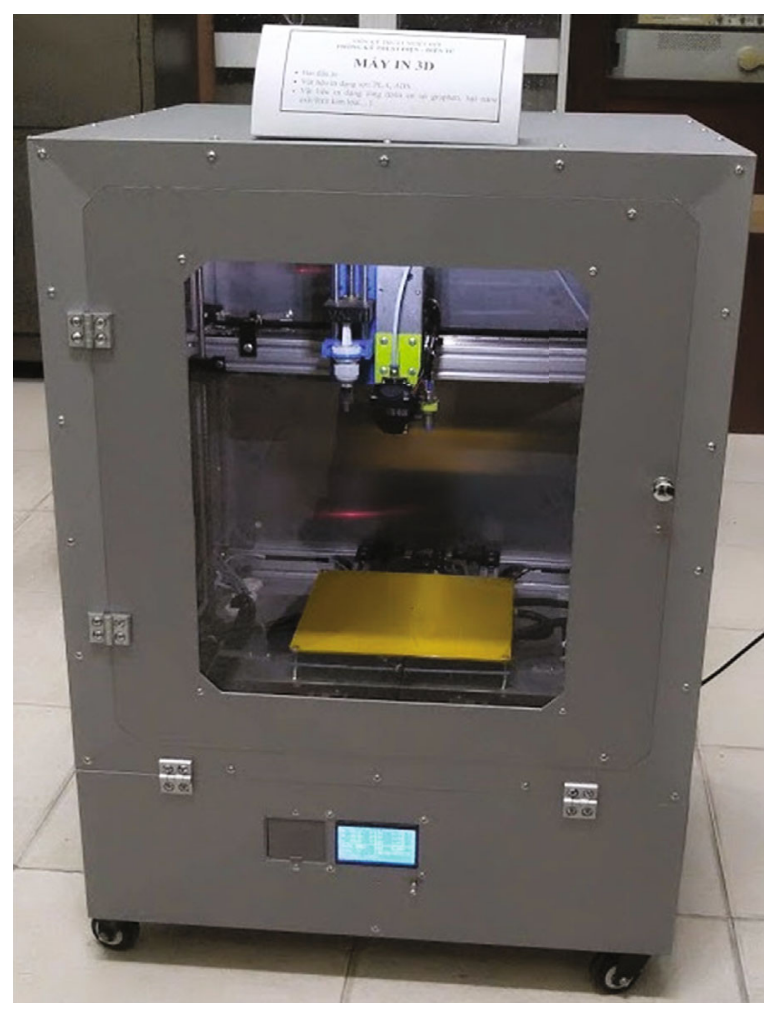

FIGURE 1: 3D printing device (Vietnam).

method combined with the $3 \mathrm{D}$ printing method. The effect of the carrageenan/collagen blend on the drug release content and the drug release kinetic of allopurinol from the biocomposite films have been also assessed and discussed.

\section{Experimental}

2.1. Materials. Carrageenan was produced by Sigma-Aldrich in powder form. $\kappa$-Carrageenan is predominant and $\lambda$-carrageenan is small. Allopurinol was provided by Sigma-Aldrich in powder form, with a purity of $\geq 98 \%$. Collagen was extracted from fresh water carp fish scales according to the procedure presented in Ref. [46]. The main characteristics of the extracted collagen are collagen type I, a fibril with a molecular weight of $116 \mathrm{kDa}$, containing 18 amino acids, with an amino acid sequence similar to that of hypothetical protein cypCar-00045321, partial (Cyprinus carpio) (NCBIprot database). Other chemicals including $\mathrm{CH}_{3} \mathrm{COOH}$, glucose, $\mathrm{KCl}, \mathrm{NaOH}, \mathrm{KH}_{2} \mathrm{PO}_{4}, \mathrm{HCl}, \mathrm{H}_{2} \mathrm{SO}_{4}$, and $\mathrm{NaCl}$ are analysis chemicals and used as received.

2.2. Fabrication of Carrageenan/Collagen/Allopurinol Biocomposite Films. The procedure for the fabrication of carrageenan/collagen/allopurinol biocomposite films is as follows: firstly, carrageenan solution is prepared by dissolving $0.1 \mathrm{~g}$ of carrageenan in $10 \mathrm{~mL}$ of distilled water. The solution was stirred on a magnetic stirrer (IKA, Germany) for $30 \mathrm{mins}$ at $80^{\circ} \mathrm{C}$ with a speed of $400 \mathrm{rpm}$ before cooling to $50^{\circ} \mathrm{C}$. The $\mathrm{KCl}$ solution ( $1 \mathrm{mg}$ of $\mathrm{KCl}$ in $0.5 \mathrm{~mL}$ of distilled water) was dropped into the carrageenan solution, and this solution was stirred continuously at $50^{\circ} \mathrm{C}$ for $30 \mathrm{mins}$ to obtain 


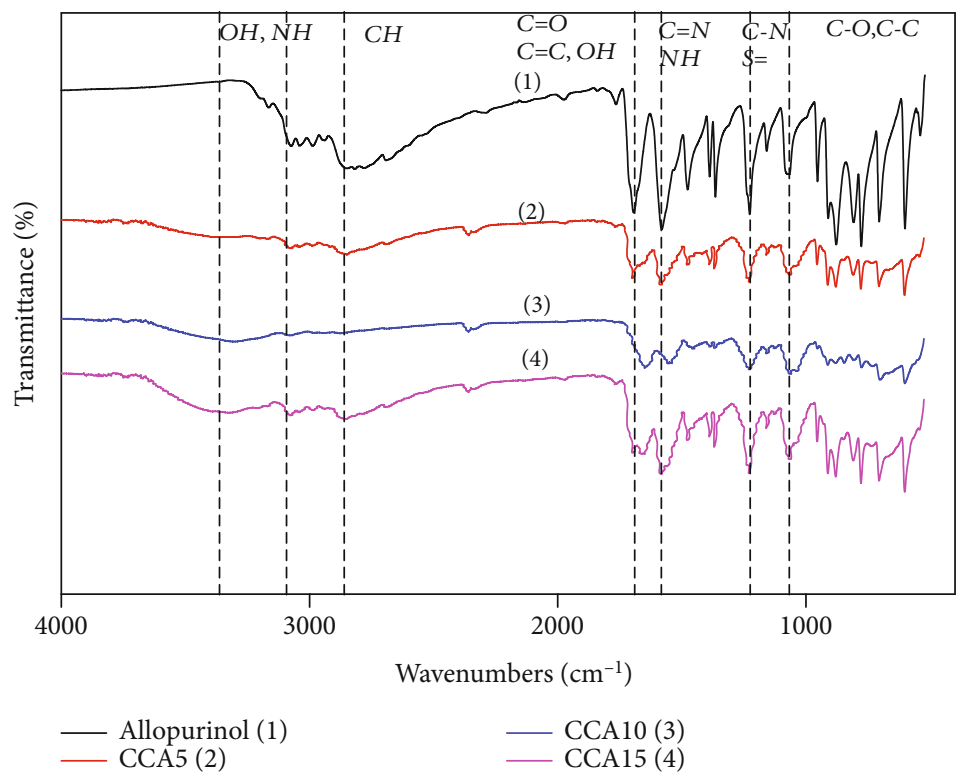

FIgURE 2: IR spectra of allopurinol and CCA biocomposite films.

TABLE 1: Vibrations of some functional groups of allopurinol, collagen, carrageenan, and CCA biocomposite films.

\begin{tabular}{|c|c|c|c|c|c|c|c|}
\hline \multirow[b]{2}{*}{ Sample } & \multicolumn{7}{|c|}{ Vibration } \\
\hline & $\begin{array}{l}v_{\mathrm{O}-\mathrm{H}} \\
v_{\mathrm{N}-\mathrm{H}}\end{array}$ & $v_{\mathrm{C}-\mathrm{H}}$ & $v_{\mathrm{PEC}}$ & $\begin{array}{c}v_{\mathrm{C}=\mathrm{O}, \mathrm{C}=\mathrm{C}} \\
\delta_{\mathrm{O}-\mathrm{H}}\end{array}$ & $\begin{array}{l}v_{\mathrm{C}=\mathrm{N}} \\
\delta_{\mathrm{N}-\mathrm{H}}\end{array}$ & $v_{\mathrm{C}-\mathrm{N}, \mathrm{S}=\mathrm{O}}$ & $v_{\mathrm{C}-\mathrm{O}, \mathrm{C}-\mathrm{C}}$ \\
\hline Allopurinol & $\begin{array}{l}3165 \\
3074\end{array}$ & $\begin{array}{l}2917 \\
2897\end{array}$ & - & $\begin{array}{l}1765 \\
1694\end{array}$ & 1580 & 1226 & 1069 \\
\hline Collagen & $\begin{array}{l}3277 \\
3072\end{array}$ & 2934 & - & 1630 & 1538 & 1238 & 1081 \\
\hline Carrageenan & 3391 & 2930 & - & 1633 & - & 1223 & 1036 \\
\hline CCA5 & $\begin{array}{l}3308 \\
3079\end{array}$ & $\begin{array}{l}2946 \\
2865\end{array}$ & 1973 & $\begin{array}{l}1758 \\
1649\end{array}$ & 1555 & 1227 & 1065 \\
\hline CCA10 & $\begin{array}{l}3328 \\
3076\end{array}$ & $\begin{array}{l}2992 \\
2848\end{array}$ & 1963 & $\begin{array}{l}1766 \\
1697\end{array}$ & 1583 & 1228 & 1066 \\
\hline CCA15 & $\begin{array}{l}3324 \\
3076\end{array}$ & $\begin{array}{l}2936 \\
2851\end{array}$ & 1968 & $\begin{array}{l}1766 \\
1696\end{array}$ & 1581 & 1227 & 1066 \\
\hline
\end{tabular}

carrageenan gel. Then, the carrageenan gel was cooled to room temperature $\left(25 \pm 0.1^{\circ} \mathrm{C}\right)$ (solution $\mathrm{A}$ ).

At the same time, the collagen solution was prepared by dissolving $1 \mathrm{~g}$ of collagen in $20 \mathrm{~mL}$ of $1 \%$ acetic acid solution on a magnetic stirrer (IKA, Germany). After dissolving completely, glucose solution $(0.01 \mathrm{~g}$ of glucose in $0.5 \mathrm{~mL}$ of distilled water) was dropped into the collagen solution to create cross-linked collagen (solution B).

Allopurinol with different weights was dissolved in $5 \mathrm{~mL}$ of $0.1 \mathrm{M} \mathrm{NaOH}$ solution (solution $\mathrm{C}$ ) on a magnetic stirrer (IKA, Germany).

After preparing the solutions, solution C was slowly dropped into solution B which was homogenized at 20000 rpm using the T25 digital ULTRA-TURRAX ${ }^{\circledR}$ (IKA, Germany) to obtain the $\mathrm{BC}$ solution mixture. Next, a solution was continuously dropped into the BC solution mixture in homogenization to obtain a carrageenan/collagen/allopurinol mixture. After that, the mixture was centrifuged using a Hettich EBA 21 (Germany) and rinsed several times with dis- tilled water to remove residual ions in the sample. The carrageenan/collagen/allopurinol was obtained in a gel. The carrageenan/collagen/allopurinol gel was injected into the cylinder of a 3D printing device and considered as a 3D printing ink material.

The $3 \mathrm{D}$ printing process was carried out on a $3 \mathrm{D}$ printing device (Figure 1) (produced by the Institute for Tropical Technology, Vietnam Academy of Science and Technology, Vietnam) at room temperature. Firstly, the shape of the sample was designed on the Solid Works software with a $3 \mathrm{D}$ size of $80 \mathrm{~mm} \times 80 \mathrm{~mm} \times 0.4 \mathrm{~mm}$. The designed sample was changed to .stl format and calculated to create a G-code script on the KISSlicer software. The thickness of the printing layer is $0.2 \mathrm{~mm}$, the width of the print line is $0.6 \mathrm{~mm}$, the area of the filling is $33 \%$, the speed of print is $10 \mathrm{~mm} / \mathrm{s}$, and the temperature of print table is room temperature. In the printing process, the carrageenan/collagen/allopurinol gel was pushed out and printed on a glass sheet. After the printing process, the film was left to dry naturally at room temperature. The 


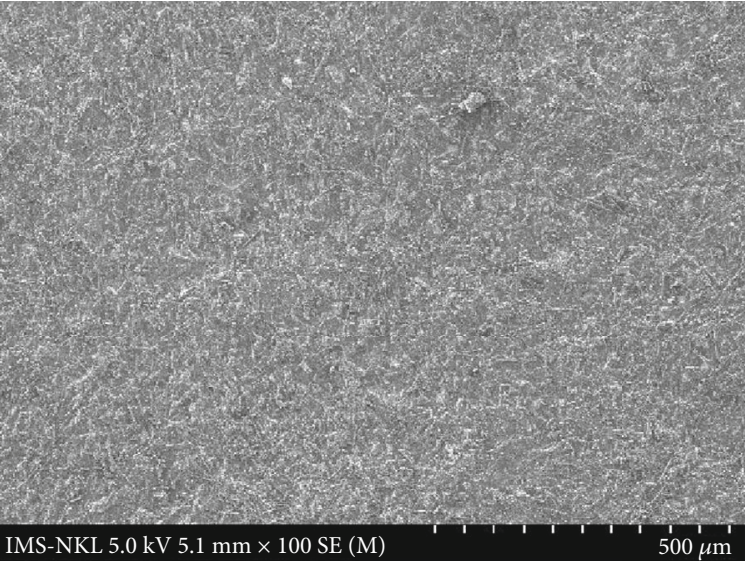

(a)

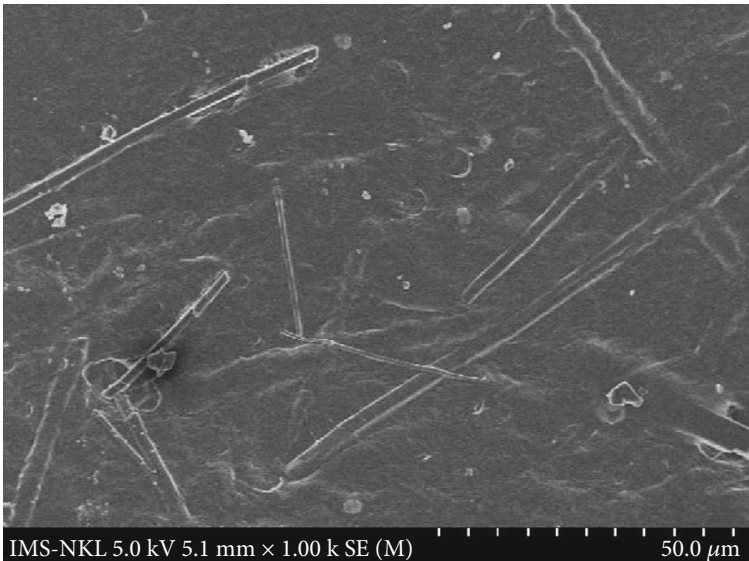

(c)

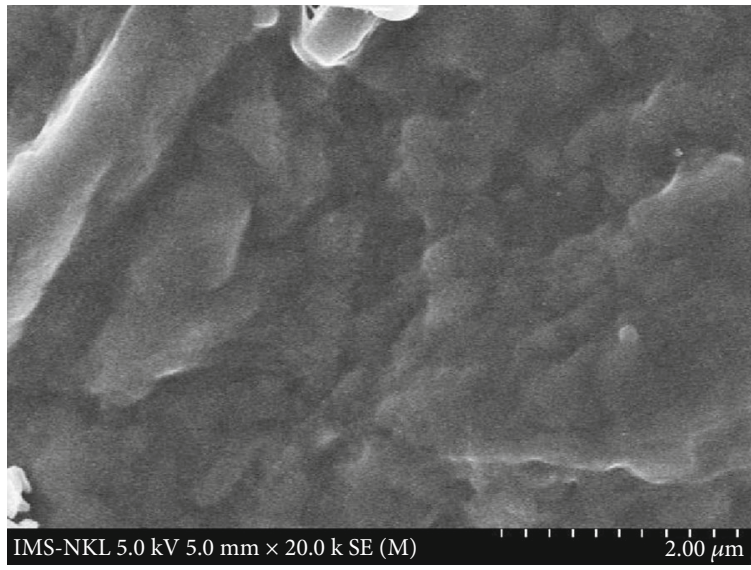

(e)

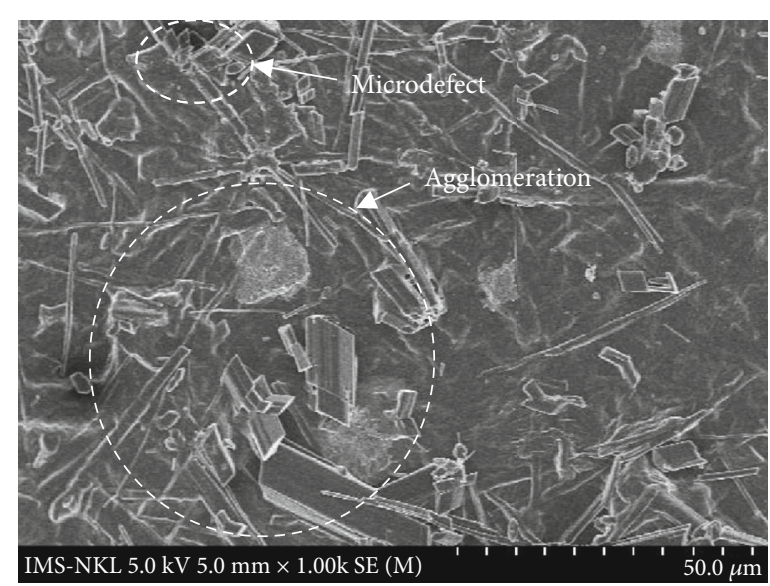

(b)

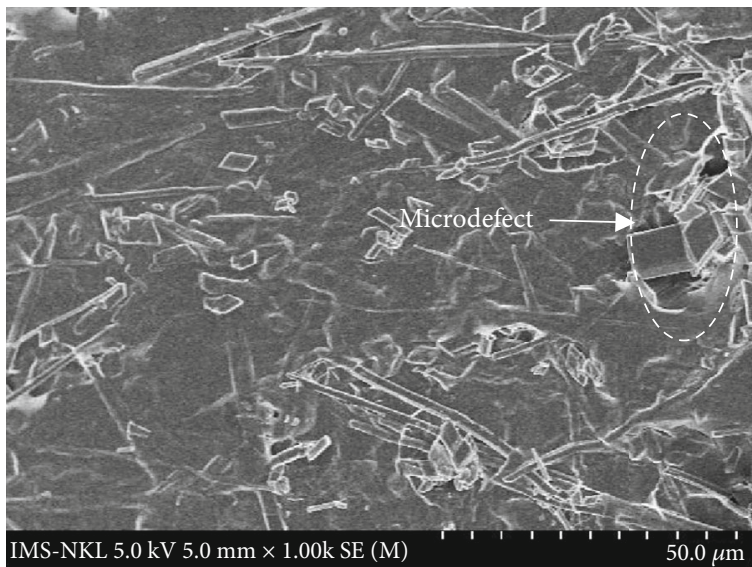

(d)

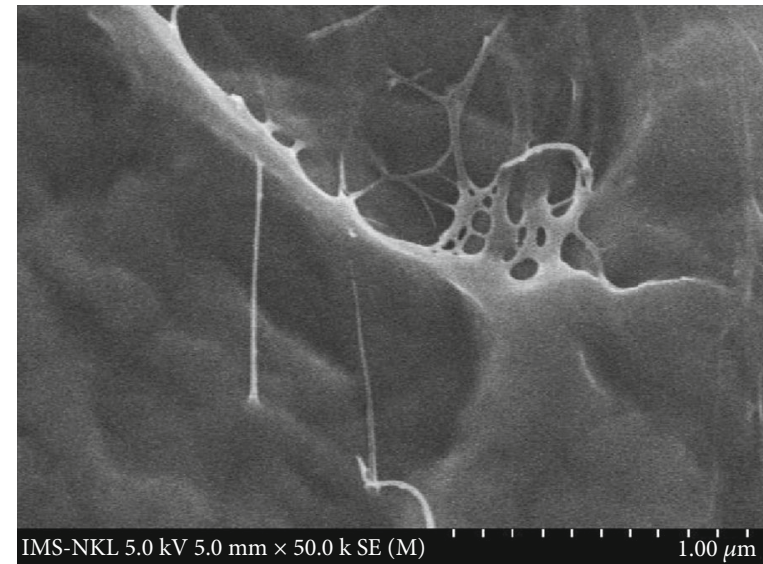

(f)

FIgURE 3: FESEM images of CCA biocomposite films: CCA15 (a, b), CCA5 (c), and CCA10 (d-f).

obtained biocomposite films have a thickness of 0.2-0.3 mm. The designations of the carrageenan/collagen/allopurinol biocomposite films are CCA5, CCA10, and CCA15 corresponding to the allopurinol weights in these films of 0.05 , 0.10 , and $0.15 \mathrm{~g}$.

2.3. Characterization. The morphological observation of the biocomposite films was taken using a field emission scanning electron microscopy (FESEM) (S-4800 FESEM, Hitachi, Japan). The films were coated with $\mathrm{Pt}$ to increase conduction. The infrared (IR) spectra of the films were recorded using a Nicolet iS10 FTIR Spectrometer (Thermo Fisher Scientific, USA) in the wavenumber range of $400 \mathrm{~cm}^{-1}-4000 \mathrm{~cm}^{-1}$, with a resolution of $8 \mathrm{~cm}^{-1}$ and scanning for 32 times.

The swelling behaviors of the biocomposite films in water and buffer solutions were also performed using a previously 

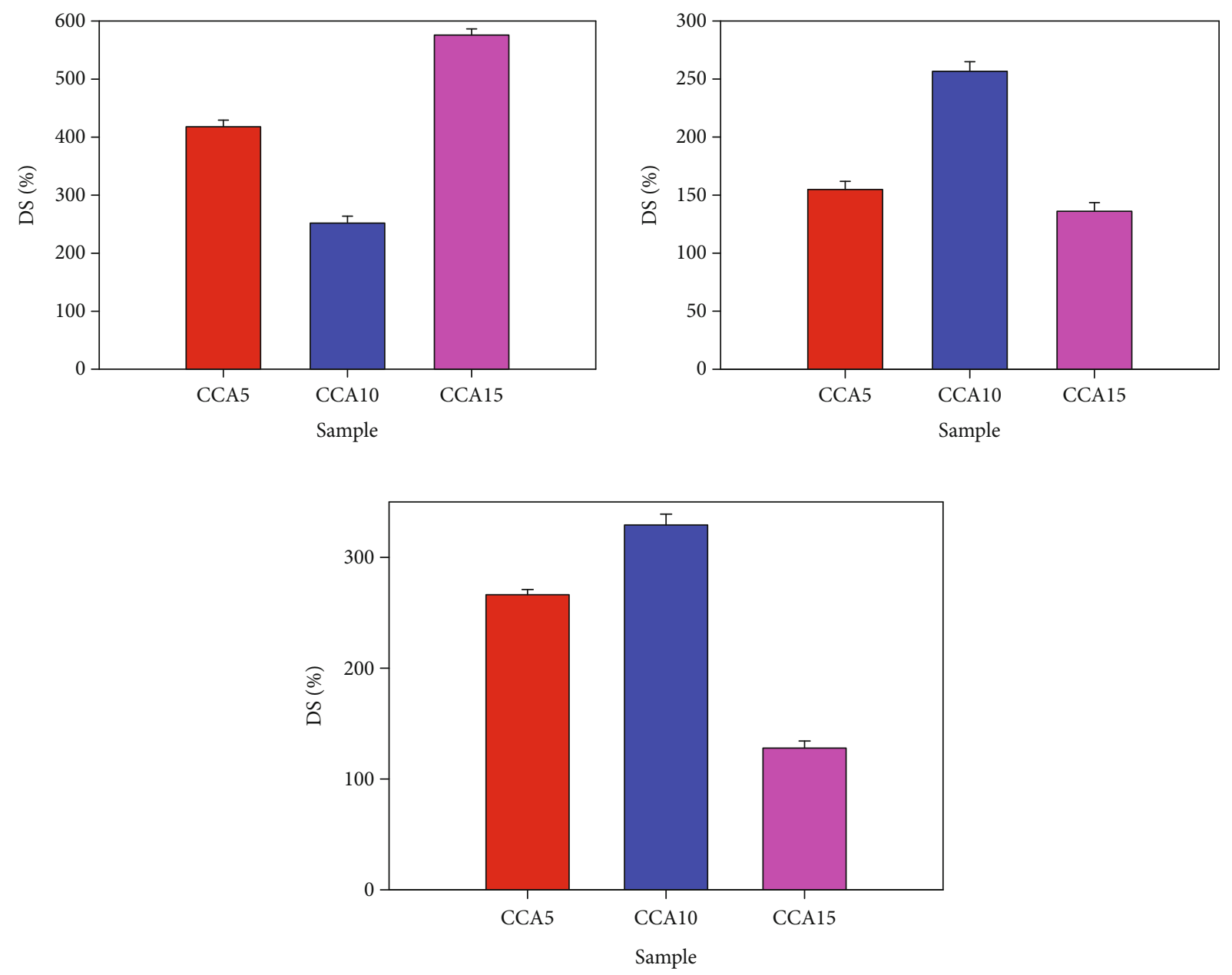

FIgURE 4: DS of CCA biocomposite films in distilled water and in $\mathrm{pH} 2.0$ and $\mathrm{pH} 7.4$ solutions.

reported procedure [42]. The degree of swelling (DS) (\%) of the biocomposites was calculated as follows:

$$
\operatorname{DS}(\%)=\frac{\left(w_{S}-w_{I}\right)}{w_{I}} 100
$$

where $w_{I}$ and $w_{S}$ represent the initial weight of the biocomposite film (size of $1 \mathrm{~cm} \times 1 \mathrm{~cm}$ ) before immersion and the weight of the swelled biocomposite film after immersion.

2.4. Study on Drug Release Process of Allopurinol from Carrageenan/Collagen/Allopurinol Biocomposite Films. The drug release process was performed in simulated gastric fluid $(\mathrm{pH} 2.0)$ and simulated intestinal fluid ( $\mathrm{pH} 7.4) .0 .01 \mathrm{~g}$ of the biocomposite film was weighted exactly and added into 200 $\mathrm{mL}$ of buffer solution at $37 \pm 0.1^{\circ} \mathrm{C}$. The mixture was stirred continuously for $8 \mathrm{hrs}$ at a speed of $400 \mathrm{rpm}$. After $1 \mathrm{hr}, 5$ $\mathrm{mL}$ of the solution was withdrawn and $5 \mathrm{~mL}$ of fresh buffer was introduced into the mixture to keep a constant volume. The ultraviolet-visible (UV-Vis) spectrum of the withdrawn solution was taken using a UV-Vis spectrophotometer (Libra
S80, Biochrom, UK) at the wavelength range of $200 \mathrm{~nm}-400$ $\mathrm{nm}$. The drug release amount was calculated using

$$
\text { Allopurinol release amount }(\%)=\frac{m_{t}}{m_{0}} 100 \text {, }
$$

where $m_{t}$ and $m_{0}$ stand for drug amounts released at $t$ time and initial time, respectively. $m_{t}$ was calculated based on the optical density value of the solution obtained at $t$ time and calibration equations of allopurinol in $\mathrm{pH} 2$ and $\mathrm{pH} 7.4$ solutions. The experiments were done three times, and the drug release amount was analyzed by Student's $t$-test comparison method for two independent groups. Significant differences were defined at $p<0.05$.

\section{Results and Discussion}

3.1. IR Spectra of Carrageenan/Collagen/Allopurinol Biocomposite Films. The IR spectra of allopurinol and CCA biocomposite films are presented in Figure 2. The vibrations of the $\mathrm{N}-\mathrm{H}, \mathrm{C}-\mathrm{H}, \mathrm{C}=\mathrm{O}, \mathrm{C}=\mathrm{N}, \mathrm{C}-\mathrm{N}$, and $\mathrm{C}-\mathrm{O}$ groups of allopurinol were found at wavenumbers of 3165 and 3074, 
2917 and 2897, 1765 and 1694,1580,1226, and $1069 \mathrm{~cm}^{-1}$, respectively. The carrageenan/collagen blend has an effect on the functional groups of allopurinol. In the biocomposite films, the vibrations of the $\mathrm{N}-\mathrm{H}, \mathrm{C}=\mathrm{O}, \mathrm{C}=\mathrm{N}$, and $\mathrm{C}-\mathrm{N}$ groups were shifted slightly (Table 1) because of the formation of hydrogen bonding between amide groups of collagen, hydroxyl groups of carrageenan, and the functional groups of allopurinol [8]. Moreover, a new peak with weak intensity appeared at about $1963-1973 \mathrm{~cm}^{-1}$ and was assigned to the vibration of the polyelectrolyte complex between the protonated amine groups of collagen and the sulfate groups of carrageenan $[30,33]$. As the allopurinol content increased, the intensity of the peaks characterized for vibrations of the $\mathrm{C}=\mathrm{O}$ and $\mathrm{C}=\mathrm{C}$ groups of allopurinol also increased.

\subsection{Morphology of Carrageenan/Collagen/Allopurinol} Biocomposite Films. The FESEM images of the CCA biocomposite films are shown in Figure 3. At the magnification of 100 times, the biocomposite films have a microstructure with a relatively regular surface (Figure $3(\mathrm{a})$ ). At higher magnification (1000 times) (Figures 3(b)-3(d)), the biocomposite films exhibit a heterogeneous structure with the dispersion of crystal allopurinol at the block form ( 5 to $50 \mu \mathrm{m}$ ) in the carrageenan/collagen blend. Allopurinol can disperse more regularly in a polymer matrix of samples containing a lower content of allopurinol (Figures 3(b) and 3(c)) and a higher content of agglomerate (Figure 3(d)). There are some microdefects in the CCA10 and CCA15 samples (Figure 3(c) and 3(d)). By observing the FESEM images of the CCA10 sample at a higher magnification (Figures 3(e) and 3(f)), it can be seen that the biocomposite film has a homogenous structure, suggesting that carrageenan and collagen were compatible together.

\subsection{Swelling Behavior of Carrageenan/Collagen/Allopurinol} Biocomposite Films. The degree of swelling of the CCA biocomposite films in different solutions is presented in Figure 4. It can be seen that CCA biocomposite films were swelled in distilled water and in pH2.0 and pH7.4 buffer solutions due to the swelling of carrageenan and collagen in the films $[9,27,28]$. The films prepared by 3D printing method have a less compact structure as compared to those prepared by solution casting, resulting these films to absorb water more easily. The degree of swelling of the CCA films in the above solutions is ordered in distilled water $>\mathrm{pH} 7.4$ solution $>\mathrm{pH} 2.0$ solution. In $\mathrm{pH} 2.0$ and $\mathrm{pH} 7.4$ solutions, the films were swelled and disintegrated due to the effect of ions in solutions, therefore, the degree of swelling of these films in pH 2.0 and pH 7.4 solutions is lower than that in distilled water. In pH 2.0 solution, a part of collagen can be dissolved into the solution, leading to the decrease in degree of swelling of films. The content of allopurinol has an unsystematic effect on the degree of swelling of CCA biocomposite films. This may be explained by the agglomeration of allopurinol at high content, thus, the microdefects are formed inside the biocomposite films, resulting in the penetration of water into the films easier. The CCA15 biocomposite film has a high degree of swelling in distilled water while a low degree of swelling in $\mathrm{pH} 2.0$ and $\mathrm{pH} 7.4$ solutions as indicated in Figure 4. This result may be due to the strong swelling abil-
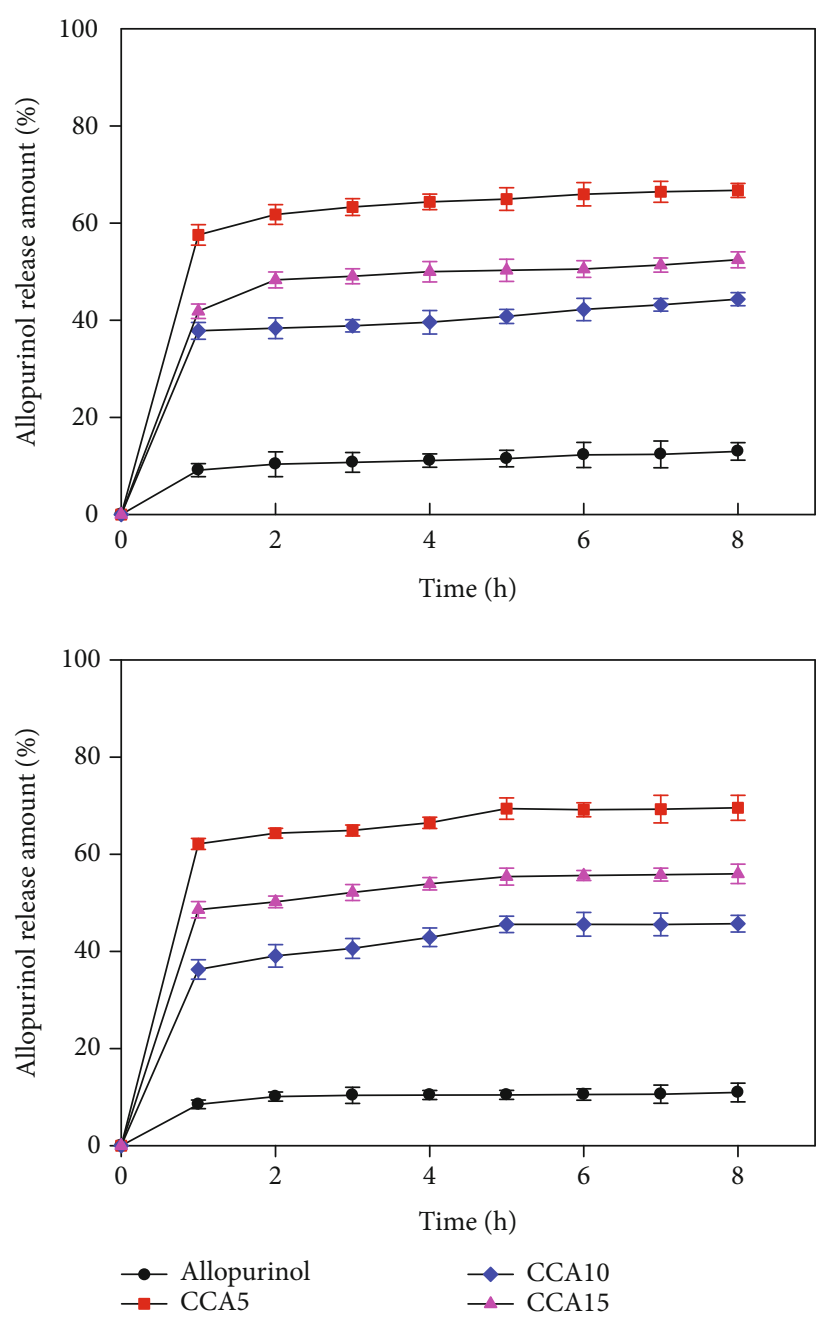

FIGURE 5: Graphs reflecting the amount of allopurinol released from CCA biocomposite films vs. time in $\mathrm{pH} 2.0$ and $\mathrm{pH} 7.4$ solutions.

ity of carrageenan in water (about 6.5 times its weight) as reported by Distantina et al. [47].

3.4. Drug Release Process of Allopurinol from Carrageenan/Collagen/Allopurinol Biocomposite Films. The release amount of allopurinol from crystal allopurinol and CCA biocomposite films in different buffer solutions is graphed in Figure 5. By comparing two groups (group 1: allopurinol release amount in the $\mathrm{pH} 2.0$ solution; group 2: allopurinol release amount in the $\mathrm{pH} 7.4$ solution), $p$ is 0.122 , confirming that there is no statistically significant difference between the two tested groups. By observing both graphs, a large part of crystal allopurinol was dissolved in the first hour of testing with the allopurinol release amount of $8.488 \%$ and 9.135\% in $\mathrm{pH} 7.4$ and $\mathrm{pH} 2.0$ solutions, respectively. The amount of released allopurinol was increased 2 to $3 \%$ depending on the $\mathrm{pH}$ of the solution in the next hours. For example, after $8 \mathrm{hrs}$ of testing, the amount of allopurinol released in the $\mathrm{pH} 7.4$ and $\mathrm{pH} 2.0$ solutions reached $10.953 \%$ and $12.987 \%$, respectively [36]. In an acidic environment, the N-H groups of allopurinol can be protonated, 
TABLE 2: $R^{2}, p$, and $n$ of kinetic models reflecting the release process of allopurinol from CCA biocomposite films to $\mathrm{pH} 2.0$ and $\mathrm{pH} 7.4$ solutions.

\begin{tabular}{|c|c|c|c|c|c|c|c|c|c|c|c|}
\hline \multirow{2}{*}{ Sample } & \multicolumn{2}{|c|}{$\mathrm{ZO}$} & \multicolumn{2}{|c|}{ PS } & \multicolumn{2}{|c|}{ HG } & \multicolumn{2}{|c|}{$\mathrm{HC}$} & \multicolumn{3}{|c|}{$\mathrm{KP}$} \\
\hline & $R^{2}$ & $p$ & $R^{2}$ & $p$ & $R^{2}$ & $p$ & $R^{2}$ & $p$ & $R^{2}$ & $p$ & $n$ \\
\hline \multicolumn{12}{|l|}{$\mathrm{pH} 2.0$} \\
\hline CCA5 & 0.848 & 0.001 & 0.970 & $<0.001$ & 0.929 & $<0.001$ & 0.848 & 0.001 & 0.977 & $<0.001$ & 0.581 \\
\hline CCA10 & 0.972 & $<0.001$ & 0.824 & 0.002 & 0.911 & $<0.001$ & 0.972 & $<0.001$ & 0.824 & 0.002 & 0.367 \\
\hline CCA15 & 0.707 & 0.009 & 0.828 & 0.004 & 0.803 & 0.003 & 0.707 & 0.009 & 0.874 & $<0.001$ & 0.435 \\
\hline \multicolumn{12}{|l|}{ pH 7.4} \\
\hline CCA5 & 0.883 & $<0.001$ & 0.932 & $<0.001$ & 0.931 & $<0.001$ & 0.883 & $<0.001$ & 0.942 & $<0.001$ & 0.619 \\
\hline CCA10 & 0.873 & $<0.001$ & 0.939 & $<0.001$ & 0.936 & $<0.001$ & 0.873 & $<0.001$ & 0.962 & $<0.001$ & 0.361 \\
\hline CCA15 & 0.878 & $<0.001$ & 0.970 & $<0.001$ & 0.942 & $<0.001$ & 0.878 & $<0.001$ & 0.965 & $<0.001$ & 0.483 \\
\hline
\end{tabular}

TABLE 3: SSE and MSE of kinetic models reflecting the release process of allopurinol from CCA biocomposite films to $\mathrm{pH} 2.0$ and $\mathrm{pH} 7.4$ solutions.

\begin{tabular}{lcccccccccc}
\hline \multirow{2}{*}{ Sample } & \multicolumn{2}{c}{ ZO } & \multicolumn{2}{c}{ PS } & \multicolumn{2}{c}{ HG } & \multicolumn{2}{c}{ HC } & KP \\
& & MSE & SSE & MSE & SSE & MSE & SSE & MSE & SSE & MSE \\
\hline pH 2.0 & & & & & & & & & \\
CCA5 & $1.82 .10^{-8}$ & $2.60 .10^{-9}$ & $2.87 .10^{-3}$ & $4.79 .10^{-4}$ & $1.82 .10^{-8}$ & $2.60 .10^{-9}$ & $2.02 .10^{-9}$ & $2.89 .10^{-10}$ & $3.20 .10^{-3}$ & $4.60 .10^{-4}$ \\
CCA10 & $4.14 .10^{-8}$ & $5.92 .10^{-9}$ & $4.70 .10^{-3}$ & $6.71 .10^{-4}$ & $4.14 .10^{-8}$ & $5.92 .10^{-9}$ & $4.60 .10^{-9}$ & $6.57 .10^{-10}$ & $4.70 .10^{-3}$ & $6.70 .10^{-4}$ \\
CCA15 & $1.02 .10^{-7}$ & $1.46 .10^{-8}$ & $5.49 .10^{-3}$ & $9.16 .10^{-4}$ & $1.02 .10^{-7}$ & $1.46 .10^{-8}$ & $1.14 .10^{-8}$ & $1.62 .10^{-9}$ & $6.33 .10^{-3}$ & $9.04 .10^{-4}$ \\
\hline pH 7.4 & & & & & & & & & \\
CCA5 & $1.63 .10^{-8}$ & $2.33 .10^{-9}$ & $7.86 .10^{-3}$ & $1.31 .10^{-3}$ & $1.63 .10^{-8}$ & $2.33 .10^{-9}$ & $1.81 .10^{-9}$ & $2.59 .10^{-10}$ & $2.52 .10^{-3}$ & $3.60 .10^{-4}$ \\
CCA10 & $7.68 .10^{-8}$ & $1.10 .10^{-8}$ & $9.75 .10^{-3}$ & $1.62 .10^{-3}$ & $7.68 .10^{-8}$ & $1.10 .10^{-8}$ & $8.54 .10^{-9}$ & $1.22 .10^{-9}$ & $1.04 .10^{-2}$ & $1.48 .10^{-3}$ \\
CCA15 & $1.33 .10^{-7}$ & $1.19 .10^{-8}$ & $3.40 .10^{-3}$ & $5.67 .10^{-4}$ & $1.33 .10^{-7}$ & $1.19 .10^{-8}$ & $1.48 .10^{-8}$ & $2.12 .10^{-9}$ & $3.85 .10^{-3}$ & $5.50 .10^{-4}$ \\
\hline
\end{tabular}

making it easier to improve the dissolution of allopurinol in the $\mathrm{pH} 2.0$ solution than in the $\mathrm{pH} 7.4$ solution.

The release process of allopurinol from CCA biocomposite films in buffer solutions is similar to the dissolution of crystal allopurinol as mentioned above. This is the fast release in the first hour and the slow release in the following hours. There is a statistically significant difference between the amount of allopurinol released from crystal allopurinol and from CCA biocomposite films $(p=0.005)$ or the amount of allopurinol released from CCA5, CCA10, or CCA15 samples in $\mathrm{pH} 2.0$ and $\mathrm{pH} 7.4$ solutions $(p=0.005)$. The fast release of allopurinol from the CCA films for the first hour of testing in pH 2.0 and pH 7.4 solutions may be due to the swelling and disintegration of the biopolymer matrix (as above discussed), which caused a part of the drug placed on the surface of biocomposite films to be released into the solutions. In the following hours, the release of allopurinol from the CCA films was controlled thanks to the interactions between polymers and drug and the drug was diffused through the polymer matrix into the solution $[36,48]$.

In the pH 2.0 solution, the amount of allopurinol which was released from CCA biocomposite films reached $37.811 \%-57.579 \%$ after $1 \mathrm{hr}$ of testing and $44.356 \%-66.751 \%$ after $8 \mathrm{hrs}$ of testing. Meanwhile, after $1 \mathrm{hr}$ and $8 \mathrm{hrs}$ of testing, the amount of allopurinol released was $36.263 \%$ $62.149 \%$ and $45.729 \%-69.594 \%$, respectively, corresponding to the release of allopurinol from CCA biocomposites in the
pH 7.4 solution. This means that the carrageenan/collagen blend contributed to the prompt dissolution of allopurinol in the neutral environment. Due to the protonation of $\mathrm{N}-\mathrm{H}$ groups in allopurinol with proton $\mathrm{H}^{+}$in an acidic solution, the bonds between polymers and drug in the neutral solution were more stable than in strong acidic conditions, leading to a better control in drug release in the $\mathrm{pH} 7.4$ solution $[48,49]$.

As compared to the allopurinol released from CCA biocomposite films which were prepared by solution casting in our previous report [50], a similar tendency can be observed, meaning that the amount of allopurinol released from composite films in the $\mathrm{pH} 7.4$ solution was higher than that in the $\mathrm{pH} 2.0$ solution. This can be explained by the protonation of amine groups of collagen, which leads to the formation of a proton layer around the film, resulting in inhibiting the diffusion of a drug through the polymer films into the solution $[23,31]$. Moreover, the swelling of biocomposite films in the $\mathrm{pH} 7.4$ solution is better than that in the $\mathrm{pH} 2.0$ solution. This also contributes to the increase in the amount of drug released from the CCA biocomposite films in the $\mathrm{pH} 7.4$ solution. However, if there is an evaluation of the effect of the preparation method on drug release from carrageenan/collagen/allopurinol films, the ionic gelation method combined with the 3D printing method exhibits a better solubility enhancement of allopurinol through a higher amount of allopurinol release as compared to the solution casting method [50]. This could be due to the better 
interaction of components as prepared by the ionic gelation method and the more regular structure of films prepared by the $3 \mathrm{D}$ printing method.

On the other hand, the allopurinol weight has an influence on the release ability of allopurinol from CCA biocomposites. The CCA5 biocomposite film has the highest drug release amount as compared to the CCA10 or CCA15 samples in both tested $\mathrm{pH}$ solutions at the same time. This may be due to the agglomeration of allopurinol in the biocomposite matrix when using high contents leading to the decrease in the diffusibility of allopurinol into solutions.

As compared to the results of allopurinol released from carrageenan/allopurinol films [51], the presence of collagen in these films also contributed in controlling allopurinol release in an acidic environment due to the formation of a proton layer as discussed above.

The kinetics of the allopurinol release process from CCA biocomposite films was fitted according to the zero order kinetic (ZO) model, the Higuchi (HG) model, the HixsonCrowell (HC) model, the Peppas-Sahlin (PS) model, and the Korsmeyer-Peppas (KP) model based on the data of the amount of drug released $[10,36,52-55]$. The $R$-square method $\left(R^{2}\right)$ and loss functions including the sum of squared error (SSE), mean squared error (MSE), and $p$ were applied to determine the most suitable kinetic model which can represent the allopurinol release process from CCA biocomposite films.

Tables 2 and 3 list the $R^{2}, p$, SSE, and MSE values of kinetic models reflecting the release process of allopurinol from CCA biocomposite films to $\mathrm{pH} 2.0$ and $\mathrm{pH} 7.4$ solutions. As can be seen, all of the above models have quite small errors (errors $<0.02$ ). This suggests that the fitting has a high accuracy. $p<0.05$ shows that there is a statistically significant difference between groups. The Korsmeyer-Peppas model has the highest $R^{2}$ which suggests that this model can fit into the release mechanism of allopurinol from CCA biocomposites into the $\mathrm{pH} 2.0$ and $\mathrm{pH} 7.4$ solutions for almost all investigated samples. These results complied with the release process of some drugs released from polymers [52, 53] because the release of allopurinol from CCA biocomposite films is a complex process which includes swelling, disintegration, protonation, diffusion, dissolution, and erosion. The values of the diffusion constant $(n)$ obtained from the KP model range from 0.361 to 0.619 depending on the component ratio of the samples and the $\mathrm{pH}$ of the solutions. This shows that the release of allopurinol from the CCA10 and CCA15 samples in both the $\mathrm{pH} 2.0$ and $\mathrm{pH} 7.4$ solutions followed a Fickian diffusion mechanism, while the drug release from the CCA5 sample is an irregular diffusion, a form that does not comply with Fick's Law [54].

\section{Conclusions}

In this study, the ionic gelation method combined with the $3 \mathrm{D}$ printing method were used to prepare carrageenan/collagen biocomposite films loading allopurinol (CCA biocomposite films). Allopurinol was dispersed and interacted with the carrageenan/collagen blend. The combination of the two methods, namely, the ionic gelation method and the $3 \mathrm{D}$ printing method, gives biocomposite films a regular microstructure and a capability to easily control the thickness and shape of CCA biocomposite films compared to the traditional method (solution casting, solvent casting). The films containing allopurinol prepared by the combined methods can enhance better the solubility of allopurinol in different buffer solutions as compared to the solution casting method. Allopurinol was diffused through polymers into solutions following the Korsmeyer-Peppas model. The carrageenan/collagen blend as well as the preparation method in this study have potential for fabricating promising polymeric drug delivery systems.

\section{Data Availability}

The data used to support the findings of this study are included within the article.

\section{Conflicts of Interest}

The authors declare no competing interests.

\section{Acknowledgments}

This research is funded by the Vietnam National Foundation for Science and Technology Development (NAFOSTED) under grant number 104.02-2017.326, period of 2018-2021.

\section{References}

[1] N. Dabholkar, S. Gorantla, T. Waghule et al., "Biodegradable microneedles fabricated with carbohydrates and proteins: revolutionary approach for transdermal drug delivery," International Journal of Biological Macromolecules, vol. 170, pp. 602-621, 2021.

[2] A. M. Ribeiro, F. Veiga, and A. Figueiras, "Biodegradable polymeric nanostructures: design and advances in oral drug delivery for neurodegenerative disorders," Nanostructures for Oral Medicine, pp. 61-86, 2017.

[3] S. A. Bhawani, A. Moheman, A. A. Yakout, and M. N. M. Ibrahim, "Chapter 10. Nanostructured biopolymers for application as drug-delivery vehicles," in Nanostructured Polymer Composites for Biomedical Applications, S. K. Swain and M. Jawaid, Eds., pp. 189-210, Elsevier, Amsterdam, The Netherlands, 2019.

[4] V. Chak, D. Kumar, and S. Visht, "A review on collagen based drug delivery systems," International Journal of Pharmacy Teaching \& Practices, vol. 4, no. 4, pp. 811-820, 2013.

[5] G. Voicu, R.-E. Geanaliu-Nicolae, A.-A. Pîrvan, E. Andronescu, and F. Iordache, "Synthesis, characterization and bioevaluation of drug-collagen hybrid materials for biomedical applications," International Journal of Pharmaceutics, vol. 510, no. 2, pp. 474-484, 2016.

[6] L. Liang, R. Ni, S. Yang, and S. Mao, "Carrageenan and its applications in drug delivery," Carbohydrate Polymers, vol. 103, pp. 1-11, 2014.

[7] E. M. Pacheco-Quito, R. Ruiz-Caro, and M. D. Veiga, "Carrageenan: drug delivery systems and other biomedical applications," Marine Drugs, vol. 18, no. 11, p. 583, 2020. 
[8] B. An, Y.-S. Lin, and B. Brodsky, "Collagen interactions: drug design and delivery," Advanced Drug Delivery Reviews, vol. 97, pp. 69-84, 2016.

[9] D.-C. Ioan, I. Rău, G. T. Tihan et al., "Piroxicam-collagenbased sponges for medical applications," International Journal of Polymer Science, vol. 2019, Article ID 6062381, 7 pages, 2019.

[10] M. G. Albu, L. Popa, M. V. Ghica, M. Leca, V. Trandafir, and I. Titorencu, "Collagen matrices for drug delivery: preparation, characterization and kinetics of release," European Cells \& Materials, vol. 16, Supplement 5, p. 1, 2008.

[11] N. Kasoju, S. S. Ali, V. K. Dubey, and U. Bora, "Exploiting the potential of collagen as a natural biomaterial in drug delivery," Journal of Proteins and Proteomics, vol. 1, no. 1, pp. 9-14, 2010.

[12] C. Kojima, T. Suehiro, K. Watanabe et al., "Doxorubicin-conjugated dendrimer/collagen hybrid gels for metastasisassociated drug delivery systems," Acta Biomaterialia, vol. 9, no. 3, pp. 5673-5680, 2013.

[13] P. Rathore, I. Arora, S. Rastogi, M. Akhtar, S. Singh, and M. Samim, "Collagen nanoparticle-mediated brain silymarin delivery: an approach for treating cerebral ischemia and reperfusion-induced brain injury," Frontiers in Neuroscience, vol. 14, article 538404, 2020.

[14] R. Constantin Barbaresso, I. Rău, R. Gabriela Zgârian, A. Meghea, and M. Violeta Ghica, "Niflumic acid-collagen delivery systems used as anti-inflammatory drugs and analgesics in dentistry," Comptes Rendus Chimie, vol. 17, no. 1, pp. 12-17, 2014.

[15] M. Obarzanek-Fojt, C. Curdy, N. Loggia et al., "Tracking immune-related cell responses to drug delivery microparticles in 3D dense collagen matrix," European Journal of Pharmaceutics and Biopharmaceutics, vol. 107, pp. 180-190, 2016.

[16] H. Jiang, G. Liang, M. Dai et al., "Preparation of doxorubicinloaded collagen-PAPBA nanoparticles and their anticancer efficacy in ovarian cancer," Annals of Translational Medicine, vol. 8, no. 14, pp. 880-880, 2020.

[17] J. McMasters and A. Panitch, "Collagen-binding nanoparticles for extracellular anti-inflammatory peptide delivery decrease platelet activation, promote endothelial migration, and suppress inflammation," Acta Biomaterialia, vol. 49, pp. 78-88, 2017.

[18] X. Fan, Y. Liang, Y. Cui et al., "Development of tilapia collagen and chitosan composite hydrogels for nanobody delivery," Colloids and Surfaces B: Biointerfaces, vol. 195, article 111261, 2020.

[19] C. Ming-Mao, C. Yu-Qing Huang, L. Yan et al., "Collagen/chitosan film containing biotinylated glycol chitosan nanoparticles for localized drug delivery," Colloids and Surfaces. B, Biointerfaces, vol. 128, pp. 339-346, 2015.

[20] I. B. Pathan, S. J. Munde, S. Shelke, W. Ambekar, and C. Mallikarjuna Setty, "Curcumin loaded fish scale collagenHPMC nanogel for wound healing application: ex-vivo and invivo evaluation," International Journal of Polymeric Materials and Polymeric Biomaterials, vol. 68, no. 4, pp. 165-174, 2019.

[21] Q. Q. Leng, Y. Li, X. L. Pang et al., "Curcumin nanoparticles incorporated in PVA/collagen composite films promote wound healing," Drug Delivery, vol. 27, no. 1, pp. 1676-1685, 2020.

[22] A. Ai, A. Behforouz, A. Ehterami et al., "Sciatic nerve regeneration with collagen type I hydrogel containing chitosan nano- particle loaded by insulin," International Journal of Polymeric Materials and Polymeric Biomaterials, vol. 68, no. 18, pp. 1133-1141, 2019.

[23] Q. Lin, Q. Huo, Y. Qin, Z. Zhao, and F. Tao, "Development of ligustrazine hydrochloride carboxymethyl chitosan and collagen microspheres: formulation optimization, characterization, and vitro release," Bioengineered, vol. 8, no. 1, pp. 55-60, 2017.

[24] F. Kianfar, M. D. Antonijevic, B. Z. Chowdhry, and J. S. Boateng, "Formulation development of a carrageenan based delivery system for buccal drug delivery using ibuprofen as a model drug," Journal of Biomaterials and Nanobiotechnology, vol. 2, no. 5, pp. 582-595, 2011.

[25] K. Tomoda, M. Asahiyama, E. Ohtsuki et al., "Preparation and properties of carrageenan microspheres containing allopurinol and local anesthetic agents for the treatment of oral mucositis," Colloids and Surfaces B: Biointerfaces, vol. 71, no. 1, pp. 27-35, 2009.

[26] M. Sathuvan, R. Thangam, M. Gajendiran et al., " $\kappa$-Carrageenan: an effective drug carrier to deliver curcumin in cancer cells and to induce apoptosis," Carbohydrate Polymers, vol. 160, pp. 184-193, 2017.

[27] M. A. R. D. Fauzi, P. Pudjiastuti, E. Hendradi, R. T. Widodo, and M. C. I. M. Amin, "Characterization, disintegration, and dissolution analyses of carrageenan-based hard-shell capsules cross-linked with maltodextrin as a potential alternative drug delivery system," International Journal of Polymer Science, vol. 2020, Article ID 3565931, 7 pages, 2020.

[28] H. Hezaveh, I. I. Muhamad, I. Noshadi, L. Shu Fen, and N. Ngadi, "Swelling behaviour and controlled drug release from cross-linked $\kappa$-carrageenan/ $\mathrm{NaCMC}$ hydrogel by diffusion mechanism," Journal of Microencapsulation, vol. 29, no. 4, pp. 368-379, 2012.

[29] J. S. Varghese, N. Chellappa, and N. N. Fathima, "Gelatin-carrageenan hydrogels: role of pore size distribution on drug delivery process," Colloids and Surfaces B: Biointerfaces, vol. 113, pp. 346-351, 2014.

[30] J. Long, X. Yu, E. Xu et al., "In situ synthesis of new magnetite chitosan/carrageenan nanocomposites by electrostatic interactions for protein delivery applications," Carbohydrate Polymers, vol. 131, pp. 98-107, 2015.

[31] G. R. Mahdavinia, A. Mosallanezhad, M. Soleymani, and M. Sabzi, "Magnetic- and pH-responsive $\kappa$-carrageenan/chitosan complexes for controlled release of methotrexate anticancer drug," International Journal of Biological Macromolecules, vol. 97, pp. 209-217, 2017.

[32] A. Dafe, H. Etemadi, H. Zarredar, and G. R. Mahdavinia, "Development of novel carboxymethyl cellulose/ $\kappa$-carrageenan blends as an enteric delivery vehicle for probiotic bacteria," International Journal of Biological Macromolecules, vol. 97, pp. 299-307, 2017.

[33] C. Li, S. Hein, and K. Wang, "Chitosan-carrageenan polyelectrolyte complex for the delivery of protein drugs," International Scholarly Research Notices, vol. 2013, Article ID 629807, 6 pages, 2013.

[34] K. A. Alkhamis, W. M. Obeidat, and N. M. Najib, "Adsorption of allopurinol and ketotifen by chitosan," AAPS PharmSciTech, vol. 2, no. 1, pp. 27-33, 2001.

[35] J. S. Changdeo, M. Vinod, K. B. Shankar, and C. A. Rajaram, "Physicochemical characterization and solubility enhancement studies of allopurinol solid dispersions," Brazilian Journal of Pharmaceutical Sciences, vol. 47, no. 3, pp. 513-523, 2011. 
[36] N. T. Chinh, V. Q. Manh, P. T. Thuy et al., "Novel pHsensitive hydrogel beads based on carrageenan and fish scale collagen for allopurinol drug delivery," Journal of Polymers and the Environment, vol. 28, no. 6, pp. 1795-1810, 2020.

[37] D. G. Yu, "Preface," Current Drug Delivery, vol. 18, no. 1, pp. 2-3, 2021.

[38] M. Wang, D. Li, J. Li et al., "Electrospun Janus zein-PVP nanofibers provide a two-stage controlled release of poorly watersoluble drugs," Materials \& Design, vol. 196, article 109075, 2020.

[39] S. Kang, Y. He, D. G. Yu, W. Li, and K. Wang, "Drug-zein@lipid hybrid nanoparticles: electrospraying preparation and drug extended release application," Colloids and Surface BBiointerfaces, vol. 201, article 111629, 2021.

[40] L. K. Prasad and H. Smyth, "3D printing technologies for drug delivery: a review," Drug Development and Industrial Pharmacy, vol. 42, no. 7, pp. 1019-1031, 2016.

[41] F. F. P. Da Costa, E. S. Araújo, M. L. F. Nascimento, and H. P. de Oliveira, "Electrospun fibers of enteric polymer for controlled drug delivery," International Journal of Polymer Science, vol. 2015, 8 pages, 2015.

[42] M. Hashizume, Y. Murata, K. Iijima, and T. Shibata, "Drug loading and release behaviors of freestanding polysaccharide composite films," Polymer Journal, vol. 48, no. 4, pp. 545550, 2016.

[43] M. Ghavami-Lahiji, F. Shafiei, F. Najafi, and M. Erfan, "Drugloaded polymeric films as a promising tool for the treatment of periodontitis," Journal of Drug Delivery Science and Technology, vol. 52, pp. 122-129, 2019.

[44] A. Roy, S. Samanta, K. Singha et al., "Development of a thermoresponsive polymeric composite film using cross-linked $\beta$-cyclodextrin embedded with carbon quantum dots as a transdermal drug carrier," ACS Applied Bio Materials, vol. 3, no. 5, pp. 3285-3293, 2020.

[45] S. B. Mitra, "Oral sustained release drug delivery system using polymer film composites," in Polymers as Biomaterials, S. W. Shalaby, A. S. Hoffman, B. D. Ratner, and T. A. Horbett, Eds., Springer, Boston, MA, USA, 1984.

[46] N. T. Chinh, V. Q. Manh, V. Q. Trung et al., "Characterization of collagen derived from tropical freshwater carp fish scale wastes and its amino acid sequence," Natural Product Communications, vol. 14, no. 7, article 1934578X1986628, p. 12, 2019.

[47] S. Distantina, F. Fadilah, and M. Kaavessina, "Swelling behaviour of kappa carrageenan hydrogel in neutral salt solution," International Scholarly and Scientific Research \& Innovation, vol. 10, no. 8, pp. 998-1001, 2016.

[48] P. Vlieghe, T. Clerc, C. Pannecouque et al., "Synthesis of new covalently bound $\kappa$-carrageenan-AZT conjugates with improved anti-HIV activities," Journal of Medicinal Chemistry, vol. 45, no. 6, pp. 1275-1283, 2002.

[49] C. Tan, M. Arshadi, M. C. Lee et al., "A robust aqueous coreshell-shell coconut-like nanostructure for stimuli-responsive delivery of hydrophilic cargo," ACS Nano, vol. 13, no. 8, pp. 9016-9027, 2019.

[50] C. T. Nguyen, M. T. Tran, M. Q. Vu et al., "Effect of fish scale collagen on some characteristics and drug release of carrageenan/collagen/allopurinol film," Vietnam Journal of Science and Technology, vol. 57, no. 3B, pp. 1-8, 2019.

[51] N. T. Phuong, N. T. Chinh, V. Q. Manh, T. Hoang, and V. Q. Trung, "Some characteristics and allopurinol release of carrageenan/allopurinol films using polyethylene oxide as a disper- sion aid agent," Vietnam Journal of Science and Technology, vol. 58, no. 2, pp. 219-227, 2020.

[52] M. S. Amini-Fazl and H. Mobedi, "Investigation of mathematical models based on diffusion control release for paclitaxel from in-situ forming PLGA microspheres containing HSA microparticles," Materials Technology, vol. 35, no. 1, pp. 5059, 2020.

[53] A. R. Florin, B. Markus, K. Peter, W. L. Geoffrey, and C. F. Wolfgang, "Modeling of drug release from collagen matrices," Journal of Pharmaceutical Sciences, vol. 91, no. 4, pp. 964-972, 2002.

[54] R. Nanda, A. Sasmal, and P. L. Nayak, "Preparation and characterization of chitosan-polylactide composites blended with Cloisite 30B for control release of the anticancer drug paclitaxel," Carbohydrate Polymers, vol. 83, no. 2, pp. 988-994, 2011.

[55] N. A. Peppas and J. J. Sahlin, "A simple equation for the description of solute release. III. Coupling of diffusion and relaxation," International Journal of Pharmaceutics, vol. 57, no. 2, pp. 169-172, 1989. 